\title{
Glomerular Endothelium Exhibits Enhanced Expression of Costimulatory Adhesion Molecules, CD80 and CD86, by Warm Ischemia/Reperfusion Injury in Rats
}

\author{
Shigeru Satoh, Akira Suzuki, Yasushi Asari, Mitsuru Sato, Naosuke Kojima, \\ Takeya Sato, Norihiko Tsuchiya, Kazunari Sato, Haruki Senoo, and Tetsuro Kato \\ Departments of Urology (SS, AS, YA, NT, KS, TK) and Anatomy (MS, NK, TS, HS), Akita University School of \\ Medicine, Akita, Japan
}

\begin{abstract}
SUMMARY: Recent studies suggested that the vascular endothelial cells function as a resident antigen-presenting cell (APC) in certain situations such as organ transplantation, and the ischemia/reperfusion injury, an inevitable event in organ transplantation, leads to an enhanced biosynthesis of cell adhesion molecules. We have demonstrated that the hepatic sinusoidal endothelial cells have potential ability as APCs by expressing the costimulatory adhesion molecule proteins, CD80 (B7-1) and CD86 (B7-2), of which expression was enhanced by warm ischemia/reperfusion of the rat liver. In this study, we assessed the localization of CD80, CD86, and intercellular adhesion molecule 1 in the rat kidneys and the influence of warm ischemia/reperfusion with or without a hypercreatinemic condition on the expression of these adhesion molecules in the renal tissues. Wistar male rats weighing 150 to $230 \mathrm{~g}$ were divided into group $\mathrm{A}$, receiving a sham-operation (control), group B, receiving 1-hour clamping of the left renal pedicle (temporary ischemia), and group $\mathrm{C}$, receiving right nephrectomy and 1-hour clamping of the left renal pedicle (temporary ischemia with hypercreatinemia). The left kidneys were submitted to immunohistochemical and molecular analyses sequentially for the period of 14 days. We found that CD80, CD86, and intercellular adhesion molecule 1 proteins localized on the glomerular and peritubular endothelium and were up-regulated after ischemia/reperfusion. The up-regulation of these three proteins was enhanced by the hypercreatinemic condition. The relative mRNA levels analyzed by real-time reverse transcription polymerase chain reaction showed that CD80 and CD86 expressions were constitutively observed and significantly increased for 14 days after the warm ischemia reperfusion with a peak level at Day 3 (6.7- and 20.8-fold increase for CD80 and CD86, respectively). Our results suggested that the glomerular endothelial cells will play a pivotal role as a APC by expressing CD80 and CD86 in the induction of renal tissue injury associated with the ischemia/reperfusion at renal transplantation surgery, as well as the peritubular endothelium. (Lab Invest 2002, 82:1209-1217).
\end{abstract}

I schemia/reperfusion injury occurring secondarily to organ surgery, transplantation, and trauma induces vascular endothelial cell dysfunction (Carden and Granger, 2000). Reoxygenated endothelial cells produce more oxygen radicals, but less nitric oxide, during the initial period following reperfusion (Grisham et al, 1998). The resulting imbalance between superoxide and nitric oxide in the endothelial cells leads to production and release of inflammatory mediators and enhances the biosynthesis of adhesion molecules that mediate the leukocyte-endothelial cell adhesion (Parvums, 1999). Intercellular adhesion molecule-1 (ICAM-1; CD54) plays an important role in the leukocyte-mediated tissue injury after ischemia/reperfusion (Colletti et al, 1998; Poston et al, 1999; Rabb et al, 1995).

Studies over the last decade have highlighted the importance of the costimulatory pathway of B7-CD28-

DOI: 10.1097/01.LAB.0000029620.13097.19

Received April 25, 2002.

Address reprint requests to: Dr. Tetsuro Kato, Department of Urology, Akita University, School of Medicine, Akita 010-8543, Japan. E-mail: tkato@med.akita-u.ac.jp
CTLA-4, which is essential to the proliferation of $\mathrm{T}$ cells (Sayegh and Turka, 1998). The costimulatory signal is provided by cell surface adhesion molecules after binding to their ligands on antigen-presenting cells (APCs) (Mueller et al, 1989). CD28 is a potent mediator for the costimulatory signal on T cells (Linsley et al, 1991), and CD80 (B7-1) and CD86 (B7-2) were identified as the ligands for CD28 (Azuma et al, 1993; Freeman et al, 1993). CD80 and CD86 were reported to be involved as the costimulatory signaling molecules on APCs in T-cell activation (Takada et al, 1997). Several studies reported that CD80 and CD86 expressions are up-regulated after ischemia/reperfusion injury, and the blockade of the costimulatory pathway by administering CTLA4Ig prevents the early and late consequences of ischemia/reperfusion injury (Chandraker et al, 1997; Takada et al, 1997). However, the location of increased CD80 and CD86 in the kidney after ischemia/reperfusion remains to be clarified.

Our first question was: which resident cells express CD80 and CD86 as activated APCs in each organ after warm ischemia/reperfusion injury? Recently, we showed that expressions of CD80, CD86, and ICAM-1 were up-regulated after warm ischemia/reperfusion 
injury on the surface of hepatic sinusoidal endothelial cells (Kojima et al, 2001). Hepatic sinusoidal endothelial cells have been found to be fully efficient APCs, expressing costimulatory cell adhesion molecules. De Greef et al (2001) have found B7-1 (CD80) expression along the endothelial cells of the ascending vasa recta in the kidney soon after ischemia/reperfusion injury. Hauser et al (1997) showed de novo expression of vascular cell adhesion molecule- 1 and E-selectin and overexpression of ICAM-1 in the glomeruli in allograft recipients and patients with primary renal diseases, suggesting that glomerular endothelial cells express costimulatory adhesion molecules, as well as the endothelial cells of vasa recta, after ischemia/reperfusion injury. To investigate the location of costimulatory adhesion molecules in renal ischemia/reperfusion injury, we used dual immunofluorescence staining.

The second question was whether renal failure (hypercreatinemic conditions) enhances the expression of costimulatory adhesion molecules, because the renal allograft is transplanted in a recipient with hypercreatinemia conditions. Azuma et al (1997) suggested that early ischemia/reperfusion of a single kidney was an important antigen-independent risk factor for renal failure associated with up-regulation of ICAM-1 expression. To investigate the influence of the hypercreatinemic environment on the expression of the costimulatory molecules, we employed an experiment model using a combination of warm ischemia/ reperfusion and contralateral nephrectomy (nephrectomy-ischemia/reperfusion). This model (animals with a reduced renal mass in the hypercreatinemic condition by ischemia/reperfusion) mimicked the transplanted kidney in recipients with renal failure.

The third question was: what are the quantitative changes in enhanced costimulatory molecule messenger RNA (mRNA) after warm ischemia/reperfusion injury? Takada et al (1997) reported that B7 expression occurred within 24 hours and peaked at Day 3 during 7 days of observations after cold ischemia/reperfusion. To investigate the quantitative levels of CD80 and CD86 mRNA until Day 14 after ischemia/reperfusion, we used real-time reverse transcription polymerase chain reaction (RT-PCR).

In this study, we examined the location and enhancement of the CD80, CD86, and ICAM-1 by immunofluorescence staining and RT-PCR in the rat kidney after warm ischemia/reperfusion injury.

\section{Results}

\section{Serum Creatinine Levels after Ischemia/Reperfusion}

We quantified the serum creatinine levels after 1-hour ischemia/reperfusion (group B) and nephrectomyischemia/reperfusion (group C) for up to 14 days (Table 1). The serum creatinine level in group $C$ was significantly elevated on Days 1 and 3 and returned to the sham-operated control (group A) level on Day 7, whereas it remained virtually unchanged in group B. These results suggest that the left kidneys of group $C$ were exposed to a hypercreatinemic environment for at least 3 days.
Table 1. Change of Serum Creatinine Levels ( $\mathrm{mg} / 100$ $\mathrm{ml}$ ) after Ischemia/Reperfusion (Group B) and Nephrectomy-Ischemia/Reperfusion (Group C)

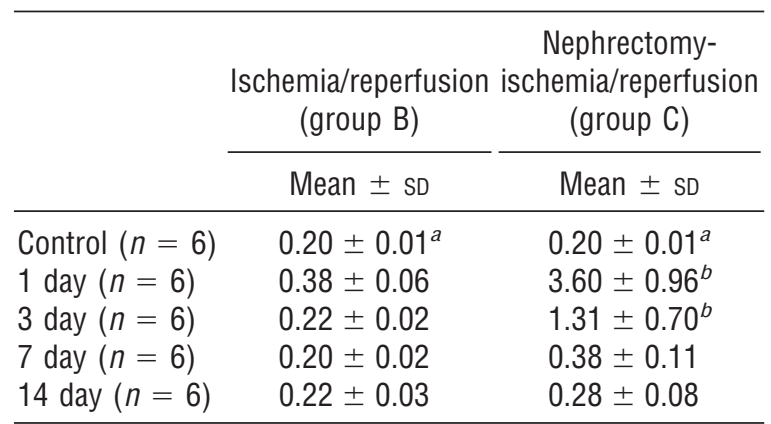

${ }^{a}$ Values from sham-operated control (group A).

${ }^{b} p<0.05$, compared with the control values.

\section{Localization of CD80, CD86, and ICAM-1 Proteins}

It was reported that CD80, CD86, and ICAM-1 proteins are expressed in the $B$ cells in the spleen tissue as well as in other APCs (Maeda et al, 1997). Therefore, the rat spleen tissue was used as a positive control to verify anti-CD80, CD86, and ICAM-1 antibodies for immunofluorescence staining. As expected, CD80, CD86, and ICAM-1 positive cells, probably APCs, were consistently detected in the marginal sinus of the spleen tissues (data not presented), suggesting that these monoclonal antibodies recognized the corresponding cell-adhesion molecules in tissue sections. The localization of CD80, CD86, and ICAM-1 proteins in the renal tissues was evaluated by indirect immunofluorescence staining using specific monoclonal antibodies against CD80, CD86, and ICAM-1, respectively. There were very weak signals derived from CD80, CD86, and ICAM-1 proteins in the endothelium of glomeruli (Fig. 1, A-a, B-a, and C-a) and peritubular microvessels (data not shown) in the sham-control rats. However, the fluorescence signals of CD80, CD86, and ICAM-1 were obvious in the endothelium of glomeruli and peritubular microvessels after ischemia/reperfusion (Fig. 1, A-b, B-b, and C-b) and nephrectomy-ischemia/reperfusion (Fig. 1, A-c, $\mathrm{B}-\mathrm{C}$, and $\mathrm{C}-\mathrm{c}$ ), suggesting an up-regulation of these cell adhesion molecule proteins. Localization of CD80, CD86, and ICAM-1 in the glomerular endothelial cells was confirmed by counterstaining with von Willebrand factor, a marker protein of the endothelial cells. The results from dual immunofluorescence staining of CD80 and von Willebrand factor (Fig. 2, a, d, and e), CD86 and von Willebrand factor (Fig. 2, b, f, and g), and ICAM-1 and von Willebrand factor (Fig. 2, c, h, and i) indicated that all CD80, CD86, and ICAM-1 were located on the endothelial cell surfaces in the glomeruli.

\section{CD80-, CD86-, and ICAM-1-Positive Glomeruli at Day 3 after Reperfusion}

Ischemia/reperfusion and nephrectomy-ischemia/ reperfusion enhanced the expression of these cell adhesion molecule proteins (Fig. 1). However, there 

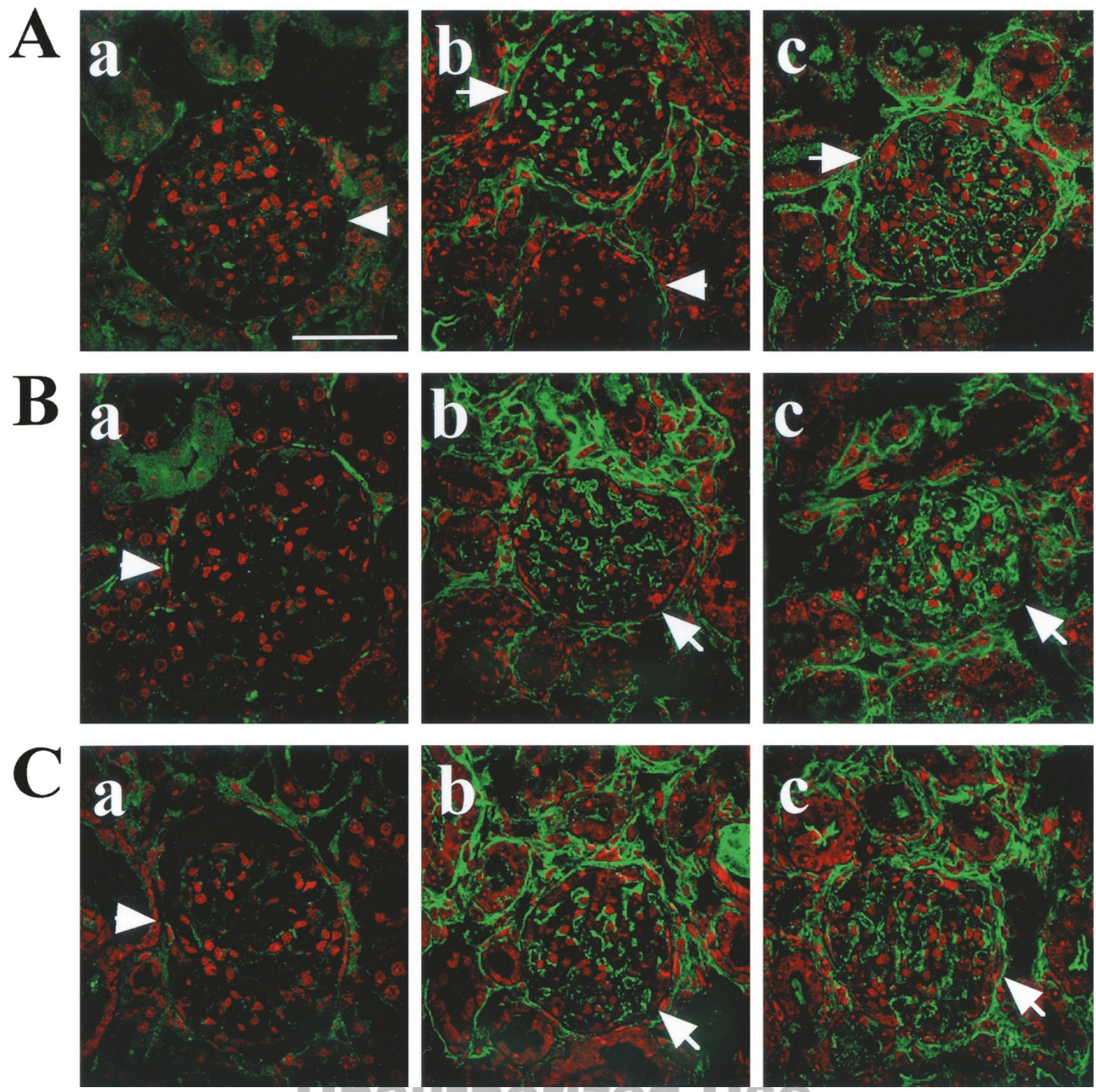

Figure 1.

Immunofluorescence staining of CD80 (A), CD86 (B), and intercellular adhesion molecule 1 (ICAM-1) (C) in the renal tissue of sham-operation (a), 3 days after 1-hour ischemia/reperfusion (b), and 3 days after 1-hour nephrectomy-ischemia/reperfusion (c). The sections were stained in green for CD80 (A), CD86 (B), and ICAM-1 (C), respectively, and counterstained with propidium iodide. The long arrows indicate the positively stained glomeruli, and the short arrows indicate the negative glomeruli. Scale bar, $50 \mu \mathrm{m}$.

was coexistence of glomeruli with and without expression of CD80, CD86, and ICAM-1, as shown in Figure $1 \mathrm{~A}-\mathrm{b}$. The positively stained glomeruli by the avidinbiotinylated horseradish peroxidase complex method were counted among at least 100 glomeruli of each kidney, as shown in Figure 3 for CD80, and the percentage of positively stained glomeruli for each of CD80, CD86, and ICAM-1 are summarized in Table 2. The percentage of positive glomeruli for each of these cell adhesion molecule proteins was less than $10 \%$ in the control group, but it was significantly increased after ischemia/reperfusion injury and further after nephrectomy-ischemia/reperfusion injury in up to $83 \%$ (Table 2). The order of staining intensity was ICAM-1 > CD86 $>$ CD80 in both the conditions. The tubular epithelium was negatively stained for these cell adhesion molecule proteins in both the proximal and distal tubules.

\section{RT-PCR Analysis of mRNA}

CD80 mRNA was hardly or very weakly detectable in the renal tissue in the control group (Fig. 4a, lane 2) but was easily detected in the renal tissue 1 day after ischemia/reperfusion (Fig. 4a, lane 3 ) by RT-PCR. The obvious CD80 mRNA expression persisted for 14 days after ischemia/reperfusion (Fig. $4 a$, lanes 3 to 6). On the other hand, CD86 and ICAM-1 mRNAs were expressed in all kidneys for the entire study period (Fig. 4, b and c). 

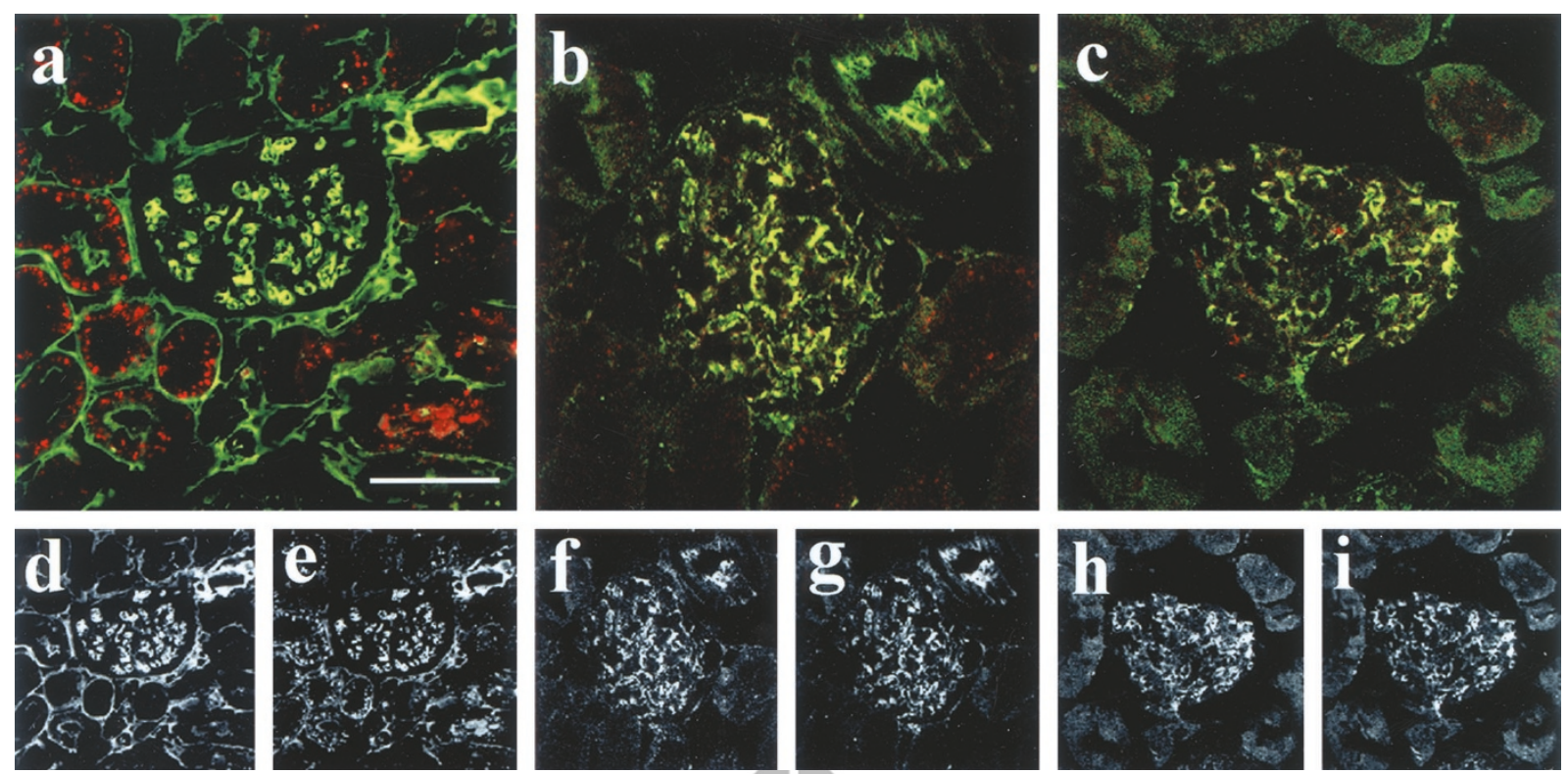

Figure 2.

Colocalization of CD80, CD86, and ICAM-1 in the glomerular endothelial cells. The sections from the left kidney 3 days after 1 -hour ischemia/reperfusion were dually stained in green for CD80 (a), CD86 (b), and ICAM-1 (c), respectively, and in red for von Willebrand factor (a, b, c). Monochromic stains showed CD80 (d), CD86 (f), ICAM-1 (h), and von Willebrand factors (e, g, i), respectively. Scale bar, $50 \mu \mathrm{m}$.

To further evaluate the quantitative mRNA levels by real-time RT-PCR, total RNA and then poly $(A)^{+}$RNA were isolated in triplicate from the renal tissues of rats in each group, and then the first-strand cDNA was generated against the poly $(A)^{+}$RNA preparation, followed by amplification for CD80 and CD86 mRNA, as well as for $\beta$-actin mRNA as an internal control. The PCR products for CD80, CD86, and $\beta$-actin were characterized by melting-curve analysis and analyzed by agarose gel electrophoresis. The CD80 and CD86 mRNA levels were normalized using the $\beta$-actin mRNA level in each poly $(A)^{+}$preparation, and the quantitative data of alteration in CD80 and CD86 mRNA levels by warm ischemia/reperfusion injury are summarized in Table 3. The relative level of CD80 mRNA to the $\beta$-actin mRNA level was approximately one third lower than that of CD86 mRNA in the control, and both CD80 and CD86 mRNA expressions were upregulated in the kidneys affected by warm ischemia/ reperfusion over the sham-operated control level. The up-regulation of CD80 and CD86 mRNA expression peaked at Day 3 (6.7- and 20.9-fold increase, respectively) and persisted at least until Day 14 (4.6- and 17.6-fold, respectively) after ischemia/reperfusion.

\section{Discussion}

The localization of CD80, CD86, and ICAM-1 on glomerular and peritubular endothelial cells was verified by dual immunostaining for each of these molecules and von Willebrand factor in the present study. The role of endothelial cells in initiating a primary $\mathrm{T}$-cell response is important in organ transplantation because the vascular endothelial cells of the allograft are the first target encountered with the recipient's immune system (Marelli-Berg et al, 1996). In vitro exper- iments showed that the vascular endothelial cells can function as resident APCs to CD4 + T cells (Maher et al, 1996; Seino et al, 1995). However, the ability of the endothelium to provide effective costimulatory signals for full T-cell activation was controversial. The endothelial cells were regarded to be incapable of inducing primary T-cell responses due to the lack of costimulatory molécule expression (Kanagawa et al, 1991; Marelli-Berg et al, 1996; Savage et al, 1991). Another study suggested that the endothelial cells express neither CD80 nor CD86 (Ma and Pober, 1998). However, CD86-dependent costimulation was demonstrated in both the human umbilical vein endothelial cells and a porcine endothelial cell line (Maher et al, 1996; Seino et al, 1995). De Greef et al (2001) found the location of B7-1 (CD80) protein in the endothelial cells of the ascending vasa recta. They mentioned that postcapillary venules, but not arteriola or capillaries, have a distinctive property of being sensitive to inflammatory mediators and are capable of presenting antigen. However, several studies revealed that some adhesion molecules induce the biosynthesis and surface expression of ligands for their adhesion molecules on the glomerular capillary endothelial cells (Brady et al, 1992; Brady and Serhan, 1992; Hauser et al, 1997; Nitta et al, 1995). Taking these and the present results together, the glomerular endothelial cells may have properties of APCs by expressing adhesion molecules for the costimulatory signal as well as the endothelial cells of the vasa recta.

A single kidney is an important antigen-independent risk factor for renal failure after ischemia/reperfusion injury associated with the up-regulation of ICAM-1 expression (Azuma et al, 1997). To investigate the influence of hypercreatinemic environment on the ex- 

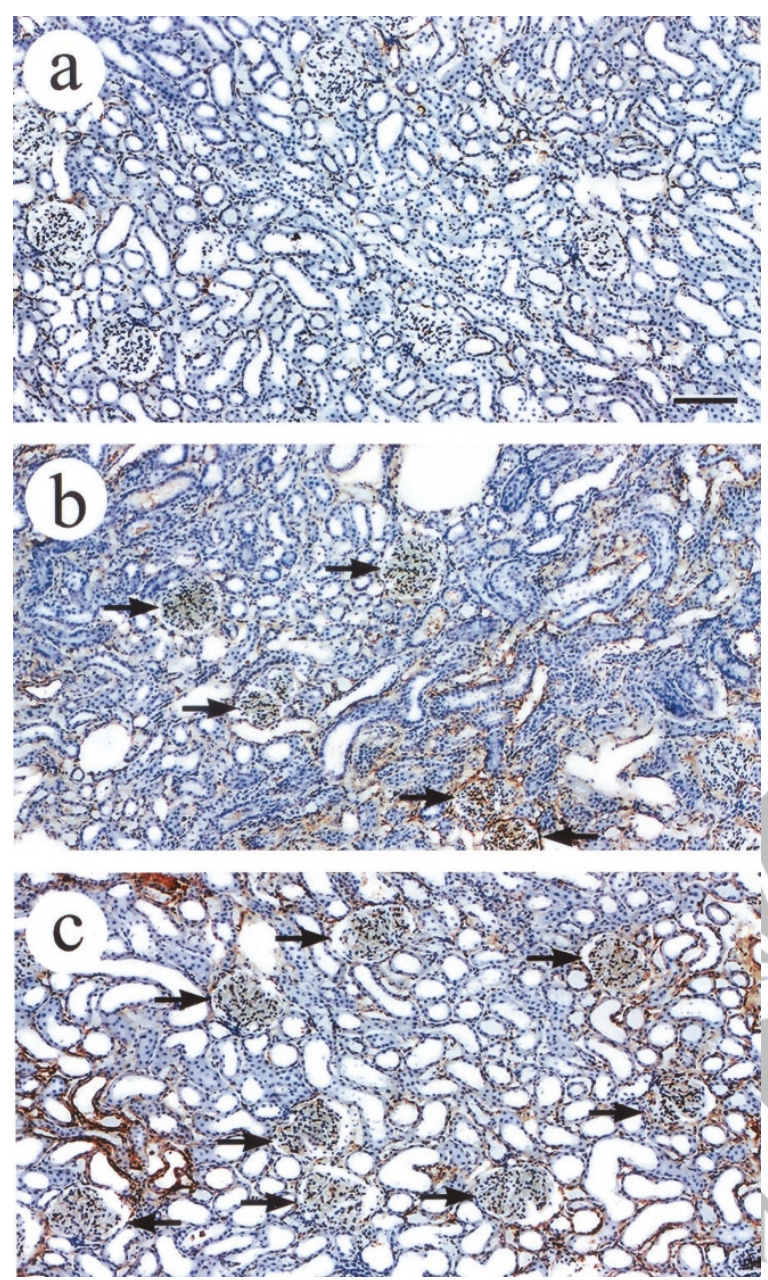

Figure 3.

Proportion of CD80 positive glomeruli in the renal cortex of sham-control rats (a), 3 days after 1 -hour ischemia/reperfusion (b), and 3 days after 1 -hour nephrectomy-ischemia/reperfusion (c). The sections were stained by avidinbiotinylated horseradish peroxidase complex. Positively stained glomeruli, as indicated by arrows, were counted among at least 100 glomeruli (see, Table 1). Scale bar, $100 \mu \mathrm{m}$.

pression of the costimulatory molecule proteins, we employed an experimental model in combination of ischemia/reperfusion and contralateral nephrectomy (nephrectomy-ischemia/reperfusion). In this study, CD80, CD86, and ICAM-1 proteins were observed in greater than $33 \%, 40 \%$, and $41 \%$ of glomeruli at Day 3 after ischemia/reperfusion, respectively. On the other hand, CD80, CD86, and ICAM-1 proteins were observed in greater than $59 \%, 76 \%$, and $83 \%$ of the glomeruli at 3 days after nephrectomy-ischemia/ reperfusion, respectively. These results suggested that the up-regulation of CD80, CD86, and ICAM-1 proteins by warm ischemia/reperfusion was further enhanced by the combination with the hypercreatinemic condition. The hypercreatinemic condition may be one of the factors that induce the biosynthesis of humoral factors such as cytokines for the upregulation of these cell adhesion molecules. Furthermore, many factors other than hypercreatinemia, such as glomerular hypertrophy, hyperfiltration, and shear stress, may influence the expression of CD80, CD86, and ICAM-1 in the remaining kidney after unilateral nephrectomy. It was suggested that glomerular hyperfiltration is one of the potential mechanisms of ICAM-1 up-regulation in diabetic glomeruli (Sugimoto et al, 1997) and renal ablation (Miyatake et al, 1998) in the rats. It may be that glomerular hyperfiltration also influences the up-regulation of CD80 and CD86 in the rat with nephrectomy-ischemia/reperfusion injury.

This study also showed that the level of CD80 mRNA expression was constitutively lower than that of CD86 mRNA. Both CD80 and CD86 mRNA were up-regulated until Day 14 and peaked at Day 3 after warm ischemia/reperfusion. The up-regulative levels of CD80 and CD86 mRNA at Day 3 after ischemia/ reperfusion compared with the sham-control levels were about 6 and 20-fold, respectively. De Greef et al (2001) found that B7-1 (CD80) but not B7-2 (CD86) was expressed on the endothelial cells of the ascending vasa recta 2 hours after reperfusion. They suggested the B7-1 protein has a key role in renal ischemia/reperfusion injury. However, the other studies showed that not only B7-1 but also B7-2 and ICAM-1 have an important role in renal ischemia/ reperfusion injury because the blockage of these molecules prevents the damage to tubular function and the glomerular filtration rate due to ischemia/reperfusion (Chandraker et al, 1997; Dragun et al, 1998; Takada et al, 1997). The up-regulation of CD80 and CD86 mRNA until Day 14 after warm ischemia/reperfusion may delay recovery of the glomerular and tubular function in renal transplantation.

An enhanced expression of ICAM-1 after ischemia/ reperfusion has been observed in several human organs such as liver (Viebahn et al, 1998), heart (Poston et al, 1999), intestine (Wyble et al, 1996), cerebral microvessels (Lindsberg et al, 1996), and kidney (Hauser et al, 1997; Schwarz et al, 2001). An enhanced expression of CD80 and CD86 has also been mentioned in patients with fulminant hepatic failure (Leifeld et al, 1999) and acute rejection of liver transplantation (Minguela et al, 2000)-but not in those with ischemia/ reperfusion injury. The up-regulation of CD80 and CD86 after ischemia/reperfusion has been investigated only in the animal experiments, including our previous (Kojima et al, 2001) and present studies. In the present study, we found that the location and enhanced expression of CD80 and CD86 by ischemia/ reperfusion injury were similar to those of ICAM-1. Further studies are needed to prove the evidence of up-regulation of the costimulatory adhesion molecules in human subjects affected by ischemia/reperfusion injury.

Recently, Vasilevko et al (2002) suggested that the CD80 and CD86 molecules can substitute for each other in the initial activation of resting CD4 positive T cells and in the maintenance of their proliferative response. However, the biologic details of CD80 and CD86 have not been fully clarified, and there have been no documents showing how many molecules are needed to produce costimulatory signals. Further studies are also needed to elucidate these issues. 
Table 2. The Percentage of CD80, CD86, and ICAM-1 Positive Glomeruli 3 Days after 1-hour Ischemia/Reperfusion and Nephrectomy-Ischemia/Reperfusion

\begin{tabular}{lccc}
\hline & \multicolumn{3}{c}{ Glomeruli expressing cell adhesion molecules (\%) } \\
\cline { 2 - 4 } & CD80: Mean & CD86: Mean & ICAM-1: \\
& \pm SD & SD & Mean \pm SD \\
\hline Control $(n=6)$ & $4.0 \pm 3.6$ & $6.3 \pm 1.5$ & $10.0 \pm 2.0$ \\
Ischemia/reperfusion $(n=6)$ & $33.7 \pm 5.1^{a}$ & $40.7 \pm 4.0^{a}$ & $41.7 \pm 2.5^{a}$ \\
Nephrectomy-ischemia/reperfusion $(n=6)$ & $59.7 \pm 4.5^{a b}$ & $76.3 \pm 1.5^{a b}$ & $83.0 \pm 2.0^{a b}$ \\
\hline
\end{tabular}

The proportions of glomeruli expressing CD80, CD86, and ICAM-1 among at least 100 glomeruli are indicated as means \pm SD from triplicated analyses.

${ }^{a} p<0.05$, compared with the control group.

${ }^{b} p<0.05$, compared with the ischemia/reperfusion group.

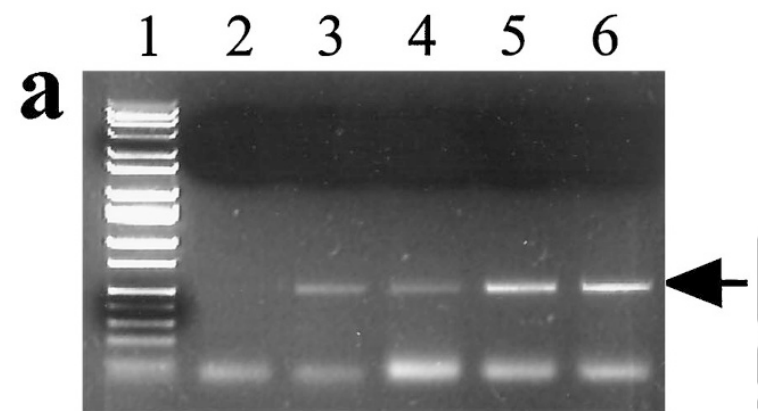

Table 3. CD80 and CD86 mRNA Levels Analyzed by Real-Time RT-PCR in the Kidneys with Ischemia/Reperfusion

b

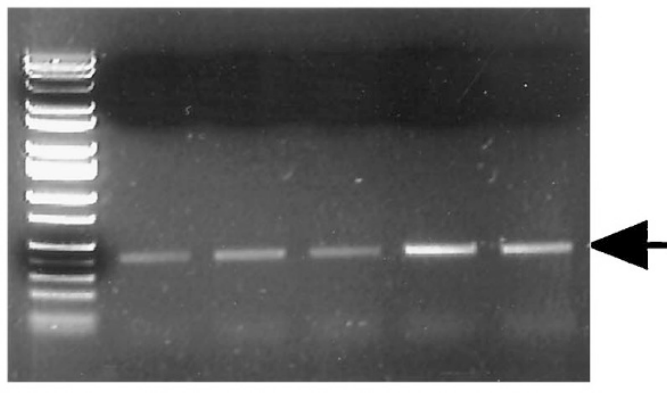

\begin{tabular}{lcc}
\hline & $\begin{array}{c}\text { CD80: Mean } \\
\pm \text { SD }\end{array}$ & $\begin{array}{c}\text { CD86: Mean } \\
\pm \text { SD }\end{array}$ \\
\hline Control $(n=3)$ & $2.5 \pm 1.6$ & $8.2 \pm 1.8$ \\
1 day $(n=3)$ & $16.1 \pm 6.2^{a}$ & $139.4 \pm 68.3$ \\
3 day $(n=3)$ & $16.8 \pm 8.8^{a}$ & $171.7 \pm 10.6^{a}$ \\
7 day $(n=3)$ & $14.8 \pm 8.1$ & $154.1 \pm 60.5^{a}$ \\
14 day $(n=3)$ & $11.5 \pm 3.2$ & $144.7 \pm 33.2^{a}$ \\
\hline
\end{tabular}

All numbers indicate mRNA levels $\left(\times 10^{-5}\right)$ normalized with the $\beta$-actin mRNA level.

c

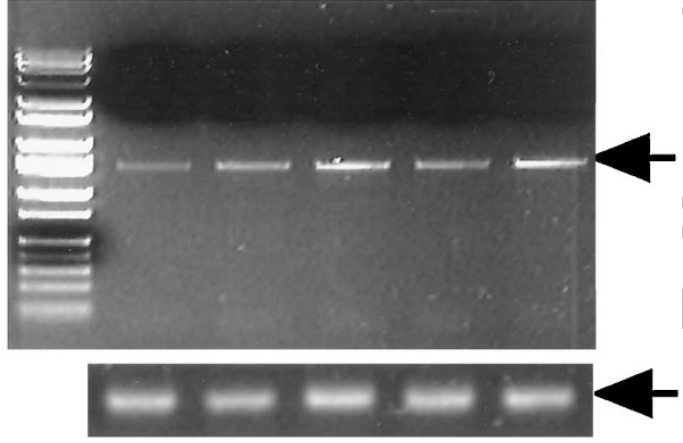

Figure 4.

CD80, CD86, and ICAM-1 mRNA levels in the renal tissue of the sham-operated control and 1-hour ischemia/reperfusion: CD80 (a), CD86 (b), ICAM-1 (c), and $\beta$-actin (d). Lane 1, DNA size markers; lane 2, sham-control; lane 3, 1 day; lane 4, 3 days; lane 5, 7 days; lane 6, 14 days after 1-hour ischemia/reperfusion. The arrows indicate the size of polymerase chain reaction (PCR) products: 520 bp for CD80, 426 bp for CD86, 1388 bp for ICAM-1, and 546 bp for $\beta$-actin.

In conclusion, the present study demonstrated that the costimulatory cell adhesion molecule proteins, CD80 and CD86, are located on the surface of the glomerular endothelial cells as well as on the endothelial cells of the vasa recta. The expression of these adhesion molecule proteins was up-regulated by warm ischemia/reperfu-
${ }^{a} p<0.05$, compared with the control values

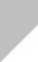

sion and further enhanced by the hypercreatinemic environment. CD80 and CD86 mRNA were constitutively expressed in the rat kidneys and further up-regulated until Day 14 after warm ischemia/reperfusion. These results suggested that the glomerular and peritubular endothelial cells play an important role as APCs by expressing CD80 and CD86 in warm ischemia/reperfusion injury similar to the hepatic sinusoidal endothelial cells (Kojima et al, 2001), and that ischemia/reperfusion and the hypercreatinemic condition can promote the early rejection and/or inflammatory reaction in renal transplantation. CD80 and CD86 might be a primary target to prevent early rejection, inflammatory reaction, and/or renal dysfunction after warm ischemia/reperfusion injury in transplant surgery.

\section{Materials and Methods}

\section{Animal Model}

Fifty-four Wistar male rats with a mean weight of $168 \mathrm{~g}$ (range, 140-190 g) were purchased from Kumagai Co. (Sendai, Japan). They were maintained under free access to conventional food and water in an airconditioned room. The rats were divided into three groups: group A, sham-operated control; group B, left renal ischemia/reperfusion (ischemia/reperfusion) group; and group C, right nephrectomy and left renal ischemia/ reperfusion (nephrectomy-ischemia/reperfusion) group. The animals were anesthetized by intraperitoneal injection of $50 \mathrm{mg}$ pentobarbital per kilogram of body weight, and then the left kidney was exposed via a left flank 
incision. In group A, the flank incision was closed without further operation, and the rats were killed 1 hour later ( $n$ $=6)$. In group $B$, the left renal pedicle was occluded with a microvascular clip for 1 hour $(n=24)$, and then the left kidney was reperfused by removing the microvascular clip. In group $\mathrm{C}$, the rats received right nephrectomy via a right flank incision followed by 1-hour ischemia operation on the left kidney as described above $(n=24)$. The flank incisions were closed after those procedures. The left kidneys, blood samples, and spleens were obtained via an abdominal midline incision under the same anesthesia at 1, 3, 7, or 14 days after reperfusion (six rats per experimental time). The protocols of the present experiments were approved by the Animal Research Committee, Akita University School of Medicine.

\section{Serum Creatinine Determination}

An $1 \mathrm{ml}$ blood sample obtained from the inferior vena cava in each rat was centrifuged at $3000 \mathrm{rpm}$ for 5 minutes, and the concentration of serum creatinine was determined using an Olympus AU800 autoanalyzer (Olympus, Tokyo, Japan). Fisher least significant difference method of ANOVA was used to assess the statistically significant difference $(p<0.05)$ among serum creatinine levels.

\section{Immunofluorescence Staining}

To avoid the intravascular blood cell reaction and to fix the renal tissues, the descending aorta was occluded proximally to the left renal artery and perfused with 10 $\mathrm{ml}$ of phosphate-buffered saline (PBS, $10 \mathrm{~mm}$ sodium phosphate, $\mathrm{pH} 7.4$, containing $0.15 \mathrm{M} \mathrm{NaCl}$ ) via a silicon tube $(0.3 \mathrm{~mm}$ diameter) catheterized from the distal aorta and then with $10 \mathrm{ml}$ of $4 \%(\mathrm{w} / \mathrm{v})$ paraformaldehyde in phosphate-buffer (PB, 100 mm sodium phosphate, $\mathrm{pH}$ 7.4). The left kidney was removed, further fixed in 4\% paraformaldehyde in PB for 24 hours, and cryoprotected in 30\% (w/v) sucrose in PB for 24 hours. The tissue specimens frozen on dry ice were cryosectioned at $10 \mu \mathrm{m}$ in thickness. The sections were mounted on 3-aminopropyltriethoxysilanecoated glass slides, air-dried for 1 hour at room temperature, washed in PBS, and postfixed in $4 \%$ paraformaldehyde in PB. After washing in PBS, the sections were incubated in $1 \%(\mathrm{w} / \mathrm{v})$ bovine serum albumin and $0.1 \%(\mathrm{w} / \mathrm{v})$ Triton X-100 (Sigma, Sigma, Saint Louis, Missouri) in PBS (PBS-T), reacted with primary antibody solution (1:100 dilution) for $60 \mathrm{~min}-$ utes, and incubated in PBS-T containing secondary antibody (1:100 dilution in PBS-T) for 30 minutes. The tissue sections were stained with fluorescein isothiocyanate (FITC)- or tetramethylrhodamine isothiocyanate (TRITC)-labeled tertiary antibody (1:100 dilution in PBS-T) for 30 minutes, and followed by washing with PBS. Antibodies used in this study were as follows: mouse monoclonal anti-rat CD80 antibody (Pharmingen, San Diego, California), mouse monoclonal antirat CD86 antibody (Pharmingen), mouse monoclonal anti-rat ICAM-1 antibody (Pharmingen), rabbit polyclonal anti-human von Willebrand factor antibody
(DAKO Co., Carpinteria, California) (Pupilli et al, 1997), rabbit anti-mouse IgG antibody (Sigma), goat antirabbit IgG antibody (Sigma), FITC-labeled anti-rabbit or anti-goat IgG antibody (Sigma), and TRITC-labeled anti-rabbit or anti-goat IgG antibody (Sigma). Several sections of each kidney stained using FITC-labeled antibody were counterstained with $0.2 \mu \mathrm{g} / \mathrm{ml}$ of propidium iodide in PBS. Fluorescence signals were analyzed using a confocal laser scanning microscope LSM-410 and LSM system soft version 3.70 (Carl Zeiss, Oberkochen, Germany).

\section{Estimation of CD80, CD86, and ICAM-1 Positive Glomeruli at Day 3 after Ischemia/Reperfusion}

Cryosections were prepared 3 days after ischemia/ reperfusion from one third of the left kidney in shamcontrol and nephrectomy-ischemia/reperfusion groups and stained for CD80, CD86, or ICAM-1, as described above, except for the use of biotinylated secondary antibody and avidin-biotinylated horseradish peroxidase complex in Vectastain ABC Kit (Vector Laboratories, Inc., Burlingame, California). Immunoreaction was visualized by peroxidase reaction with diaminobenzidine tetrahydrochloride. The number of CD80, CD86, and ICAM-1 positive glomeruli was counted among at least 100 glomeruli in each section. A significant level was defined as $p<0.05$ by Student unpaired $t$ eligible test among experimental groups.

\section{RT-PCR Analyses}

A part of the left kidney was quickly cut into small pieces and frozen and stored at $-80^{\circ} \mathrm{C}$ until further analysis. The expression of CD80, CD86, and ICAM-1 mRNA was examined by RT-PCR. Total RNA was isolated from the frozen kidney specimens $(30 \mathrm{mg})$ using RNeasy Mini-Kit (Qiagen, Hilden, Germany) and used to generate the first-strand cDNA using the 1st Strand cDNA Synthesis Kit (Boehringer Mannheim Inc., Indianapolis, Indiana). The first-strand cDNA was amplified by PCR using a pair of the specific upstream and downstream primers for CD80, CD86, ICAM-1, and $\beta$-actin, respectively, whose sequences were as follows: 5 '-CTGTCTGTCAGAGCTGACTTCCCT-3' and 5'-CCACAGGCCCTATGTAGAGGTTCT-3' for CD80, 5'-TGCTCATCTAAGCAAGGATACCCG-3' and 5'-CGACTCGTCAACACCACTGTCCTG-3' for CD86, 5'-GGGTTGGAGACTAACTGGA-3' and 5'-CCTCTGGCGGTAATAGGTG-3' for ICAM-1, and 5'-TTGTAACCAACTGGGACGATATGG-3' and 5'-ATCGGAACCGCTCATTGCC-3' for $\beta$-actin, respectively. The PCR conditions were as follows: $94^{\circ} \mathrm{C}$ for 5 minutes, followed by 35 cycles of $94^{\circ} \mathrm{C}$ for 1 minute, $57^{\circ} \mathrm{C}$ for 1 minute, and $72^{\circ} \mathrm{C}$ for 1 minute, followed by a final cycle of $72^{\circ} \mathrm{C}$ for 5 minutes. The PCR products were electrophoresed on $1 \%$ agarose gel containing 1 $\mu \mathrm{g} / \mathrm{ml}$ of ethidium bromide, and the product size was evaluated using DNA size markers. The total RNA preparation from the rat spleen tissue was used as a positive control for the detection of CD80, CD86, and ICAM-1 by RT-PCR, respectively. 
To further estimate the quantitative mRNA levels of CD80 and CD86, total RNA was extracted using TRIZOL Reagent (Life Technology Inc., Grand Island, New York) from the frozen part of kidney specimens, and then poly $(A)+$ RNA was isolated using Oligotex dT30 (Takara, Tokyo, Japan). The first-strand cDNA was generated by reverse transcription reaction using MMLV reverse transcriptase (Life Technology Inc.) and the poly (A) + RNA preparation as templates. The cDNA was amplified by real-time PCR using a LightCycler (Roche Diagnostics, Indianapolis, Indiana) with the specific upstream and downstream primers for CD80 and CD86 mRNA analysis under the following reaction condition: denaturation at $95^{\circ} \mathrm{C}$ for 2 minutes, and then 40 cycles of denaturation at $95^{\circ} \mathrm{C}$ for 0 seconds, annealing at $55^{\circ} \mathrm{C}$ for 5 seconds, and extension at $72^{\circ} \mathrm{C}$ for 15 seconds. The sequences of the upstream and downstream primers for CD80, CD86, and $\beta$-actin were as follows: 5'-GGCATTGCTGTCCTGTGATTAC-3' and 5'-ACTCAGTTATGTTGGGGGTAGG-3' for CD80; 5'-GCTCGTAGTATTTTGGCAGGACC-3' and 5'-CGGGTATCCTTGCTTAGATGAGC-3' for CD86; and 5'-TGAGAGGGAAATCGTGCGTGA-3' and 5'-TCACGCACGATTTCCCTCTCA-3' for $\beta$-actin, respectively. The product sizes for CD80 and CD86 and $\beta$-actin were $314 \mathrm{bp}, 337 \mathrm{bp}$, and $546 \mathrm{bp}$, respectively. The amplified products were analyzed by the melting curve analysis and agarose gel electrophoresis using $1.5 \%$ gel and stained using ethidium bromide. CD80 and CD86 mRNA levels were normalized with the $\beta$-actin mRNA level in each poly (A)+ RNA preparation. Significant differences were evaluated using the Student unpaired $t$ test.

\section{References}

Azuma M, Ito D, Yagita H, Okumura K, Phillips JH, Lanier LL, and Somoza C (1993). B70 antigen is a second ligand for CTLA-4 and CD28. Nature 366:76-79.

Azuma H, Nadeau K, Takada M, Mackenzie HS, and Tilney NL (1997). Cellular and molecular predictors of chronic renal dysfunction after initial ischemia/reperfusion injury of a single kidney. Transplantation 64:190-197.

Brady HR and Serhan CN (1992). Adhesion promotes transcellular leukotrience biosynthesis during neutrophilglomerular endothelial cell interactions: Inhibition by antibodies against CD18 and L-selectin. Biochem Biophys Res Commun 186:1307-1314.

Brady HR, Spertini O, Jimenez W, Brenner BM, Marsden PA, and Tedder TF (1992). Neutrophils, monocytes, and lymphocytes bind to cytokine-activated kidney glomerular endothelial cells through L-selectin (LAM-1) in vitro. J Immunol 149:2437-2444.

Carden DL and Granger DN (2000). Pathophysiology of ischemia-reperfusion injury. J Pathol 190:255-266.

Chandraker A, Takada M, Nadeau KC, Peach R, Tilney NL, and Sayegh MH (1997). CD28-b7 blockade in organ dysfunction secondary to cold ischemia/reperfusion injury. Kidney Int 52:1678-1684.

Colletti LM, Cortis A, Lukacs N, Kunkel SL, Green M, and Strieter RM (1998). Tumor necrosis factor up-regulates intercellular adhesion molecule 1 , which is important in the neutrophil-dependent lung and liver injury associated with hepatic ischemia and reperfusion in the rat. Shock 10:182191.

De Greef KE, Ysebaert DK, Dauwe S, Persy V, Vercauteren SR, Mey D, and De Broe ME (2001). Anti-B7-1 blocks mononulear cell adherence in vasa recta after ischemia. Kidney Int 60:1415-1427.

Dragun D, Tullius SG, Park JK, Maasch C, Lukitsh I, Lippoldt A, Gross V, Luft FC, and Haller H (1998). ICAM-1 antisense oligodesoxynucleotides prevent reperfusion injury and enhance immediate graft function in renal transplantation. Kidney Int 54:590-602.

Freeman GJ, Gribben JG, Boussiotis VA, Ng JW, Restivo VA Jr, Lombard LA, Gray GS, and Nadler LM (1993). Cloning of B7-2: A CTLA-4 counter-receptor that costimulates human T cell proliferation. Science 262:909-911.

Grisham MB, Granger DN, and Lefer DL (1998). Modulation of leukocyte-endothelial interactions by reactive metabolites of oxygen and nitrogen: Relevance to ischemic heart disease. Free Radic Biol Med 25:404-433.

Hauser IA, Riess R, Hausknecht B, Thuringer H, and Sterzel RB (1997). Expression of cell adhesion molecules in primary renal disease and renal allograft rejection. Nephrol Dial Transplant 12:1122-1131.

Kanagawa K, Ishikura H, Takahashi T, Tamatani T, Miyasaka M, Togashi M, Koyanagi T, and Yoshiki T (1991). Identification of ICAM-1-positive cells in the nongrafted and transplanted rat kidney-an immunohistochemical and ultrastructural study. Transplantation 52:1057-1062.

Kojima N, Sato M, Suzuki A, Sato T, Satoh S, Kato T, and Senoo H (2001). Enhanced expression of B7-1, B7-2, and intercellular adhesion molecule 1 in sinusoidal endothelial cells by warm ischemia/reperfusion injury in rat liver. Hepatology $34: 751-757$.

Leifeld L, Trautwein C, Dumoulin FL, Manns MP, Sauerbruch $T$, and Spengler $U$ (1999). Enhanced expression of CD80 (B7-1), CD86 (B7-2), and CD40 and their ligands CD28 and CD154 in fulminant hepatic failure. Am J Pathol 154:17111720.

Lindsberg PJ, Carpen O, Paetau A, Karjalainen-Lindsberg ML, and Kaste M (1996). Endothelial ICAM-1 expression associated with inflammatory cell response in human ischemic stroke. Circulation 94:939-945.

Linsley PS, Brac

Wrosmaire L, Aruffo A, Damle NK, and Ledbetter JA (1991). Binding of the B cell activation antigen B7 to CD28 costimulates T cell proliferation and interleukin 2 mRNA accumulation. J Exp Med 173:721-730.

Ma W and Pober JS (1998). Human endothelial cells effectively costimulate cytokine production by, but not differentiation of, naive CD4+ T cells. J Immunol 161:2158-2167.

Maeda K, Sato T, Azuma M, Yagita H, and Okumura K (1997). Characterization of rat CD80 and CD86 by molecular cloning and mAb. Int Immunol 9:993-1000.

Maher SE, Karmann K, Min W, Hughes CC, Pober JS, and Bothwell AL (1996). Porcine endothelial CD86 is a major costimulator of xenogeneic human T cells: cloning, sequencing, and functional expression in human endothelial cells. $\mathrm{J}$ Immunol 157:3838-3844.

Marelli-Berg FM, Hargreaves REG, Carmichael P, Dorling A, Lombardi G, and Lechler RI (1996). Major histocompatibility complex class II-expressing endothelial cells induce allospe- 
cific nonresponsiveness in native T cells. J Exp Med 183: 1603-1612.

Minguela A, Marin L, Torio A, Muro M, Garcia-Alonso AM, Moya-Quiles MR, Sanchez-Bueno F, Parrila P, and AlvarezLopez MR (2000). CD28/CTLA-4 and CD80/CD86 costimulatory molecules are mainly involved in acceptance or rejection of human liver transplant. Hum Immunol 61:658-669.

Miyatake N, Shikata K, Sugimoto $H$, Kushiro M, Shikata $Y$, Ogawa S, Hayashi Y, Miyasaka M, and Makino H (1998). Intercellular adhesion molecule 1 mediates mononuclear cell infiltration into rat glomeruli after renal ablation. Nephron 79:91-98.

Mueller DL, Jenkins MK, and Schwartz RH (1989). Clonal expansion versus functional clonal inactivation: A costimulatory signaling pathway determines the outcome of $\mathrm{T}$ cell antigen receptor occupancy. Annu Rev Immunol 7:445-480.

Nitta K, Uchida K, Miwa N, Kawashima A, Yumura W, and Nihei H (1995). Involvement of adhesion molecules in glomerular endothelial cell injury induced by PMA-stimulated lymphocytes. Endocr J 42:361-366.

Parvums DV (1999). The pathology of ischemia-reperfusion. In: Grace PA and Mathie RT, editors. Ischemia-reperfusion injury. London: Blackwell Science, 3-19.

Poston RS, Ing DJ, Ennen MP, Hoyt EG, and Robbins RC (1999). ICAM-1 affects reperfusion injury and graft function after cardiac transplantation. J Surg Res 87:25-31.

Pupilli C, Romagnani P, Lasagni L, Bellini F, Misciglia N, Emoto N, Yanagisawa M, Rizzo M, Mannelli M, and Serio M (1997). Localization of endothelin-converting enzyme-1 in human kidney. Am J Physiol 273:F749-756.

Rabb H, Mendiola CC, Saba SR, Dietz JR, Smith CW, Bonventre JV, and Ramirez G (1995). Antibodies to ICAM-1 protect kidneys in severe ischemic reperfusion injury. Biochem Biophys Res Commun 211:67-73.

Savage COS, Hughes CCW, Pepinsky RB, Wallner BP, Freedman AS, and Pober JS (1991). Endothelial cell lymphocyte function-associated antigen-3 and an unidentified ligand act in concert to provide costimulation to human peripheral blood CD4+ T cells. Cell Immunol 137:150-163.
Sayegh MH and Turka LA (1998). The role of T-cell costimulatory activation pathways in transplant rejection. $\mathrm{N}$ Engl J Med 338:1813-1821.

Schwarz C, Regele H, Steininger R, Hansmann C, Mayer G, and Oberbauer R (2001). The contribution of adhesion molecule expression in donor kidney biopsies to early allograft dysfunction. Transplantation 71:1666-1670.

Seino K, Azuma M, Bashuda H, Fukao K, Yagita H, and Okumura K (1995). CD86 (B70/B7-2) on endothelial cells co-stimulates allogeneic CD4+ T cells. Int Immunol 7:13311337.

Sugimoto H, Shikata K, Hirata K, Akiyama K, Matsuda M, Kushiro M, Shikata Y, Miyatake N, Miyasaka M, and Makino $\mathrm{H}$ (1997). Increased expression of intercellular adhesion molecule-1 (ICAM-1) in diabetic rat glomeruli: Glomerular hyperfiltration is a potential mechanism of ICAM-1 upregulation. Diabetes 46:2075-2081.

Takada M, Chandraker A, Nadeau KC, Sayegh $\mathrm{MH}$, and Tilney NK (1997). The role of the B7 costimulatory pathway in experimental cold ischemia/reperfusion injury. J Clin Invest 100:1199-1203.

Vasilevko V, Ghochikyan A, Holterman MJ, and Agadjanyan MG (2002). CD80 (B7-1) and CD86 (B7-2) are functionally equivalent in the initiation and maintenance of CD4+ T-cell proliferation after activation with suboptimal doses of PHA. DNA Cell Biol 21:137-149.

Viebahn R, Thoma M, Kinder O, Schenk M, Lauchart W, and Becker HD (1998). Analysis of intragraft adhesion molecules and their release in clinical liver transplantation: Impact or reperfusion injury. Transplant Proc 30:4257-4259.

Wyble CW, Desai-TR, Clark ET, Hynes KL, and Gewertz BL (1996). Physiologic concentrations of TNFalpha and IL-1beta released from - reperfused human intestine upregulate E-selectin and ICAM-1. J Surg Res 63:333-338.

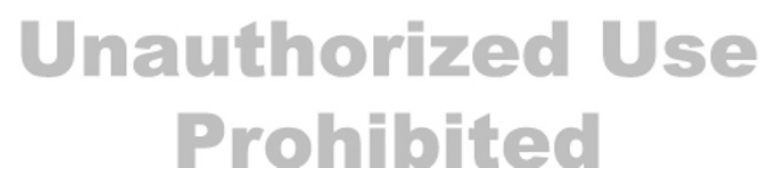

